\title{
Antioxidant Activity, Macamide B Content and Muscle Cell Protection of Maca (Lepidium meyenii) Extracted Using Ultrasonification-Assisted Extraction
}

\author{
Enkhbolor Buyanbadrakh ${ }^{1}$, Hyeong-Suk Hong ${ }^{1}$, Kang-Woo Lee ${ }^{2}$, Wen Yan Huang ${ }^{3}$, and Jun-Hyun Oh ${ }^{1 *}$ \\ ${ }^{1}$ Department of Plant and Food Sciences, Sangmyung University, Cheonan 31066, Republic of Korea \\ ${ }^{2}$ SSbiopharm Ltd., Cheonan 31056, Republic of Korea \\ ${ }^{3}$ Department of Food and Biotechnology, Korea University, Sejong 30019, Republic of Korea
}

Received: November 19, 2019 / Revised: December 2, 2019 / Accepted: December 5, 2019

\begin{abstract}
This study aims to evaluate the efficacy of the Ultrasonication-Assisted (UA) extraction on the functionality of the herbaceous biennial plant maca (Lepidium meyenii). The specific objectives include comparison of the antioxidant activities among various maca extracts, determination of the macamide $B$ content of the extracts, and in vitro evaluation of maca on cell viability and creatine kinase (CK) activity. The antioxidant activities of the water, ethanol, and UA extracts were compared by determining the total phenolic and flavonoid contents, the 2, 2-diphenyl-1-picrylhydrazyl (DPPH) radical scavenging activities, and the ferric reducing antioxidant power (FRAP) of the extracts. The macamide $B$ content of maca extracts were analyzed by HPLC. The effects of the extracts on muscle cell viability and creatine kinase activity were also determined using $\mathrm{C} 2 \mathrm{C} 12$ myoblasts. UA extraction significantly increased the total phenolic content (2.90 GAE $\mu \mathrm{g} / \mathrm{mg}, p<0.05)$, without affecting the flavonoid content. DPPH radical scavenging activity did not exhibit any statistical difference among the extracts. The ethanol and UA extracts exhibited significantly higher FRAP than the water extract $(p<0.05)$. The macamide B content of ethanol and UA extracts were 0.087 and $0.083 \mu \mathrm{g} / \mathrm{mg}$, respectively. The water and UA extracts exhibited higher C2C12 muscle cell viability than the ethanol extract, and both extracts resulted in a significantly lower CK level than the $\mathrm{H}_{2} \mathrm{O}_{2}$-treated control group. This research suggests that the maca extract can protect muscle cells and serve as an antifatigue agent under oxidative stress conditions.
\end{abstract}

Keywords: Maca, Lepidium meyenii, macamide B, ultrasonification-assisted extraction, creatine kinase

\section{Introduction}

Maca (Lepidium meyenii), an annual or biennial plant of the family of Brassicaceae, is mainly grown in Andean region of Peru where the altitude is between 3,500 and 4,500 $\mathrm{m}$ above sea level [1]. Maca is rich in nutrients and secondary metabolites with a variety of biological activities. Its main chemical compositions are polysac-

\section{*Corresponding author}

Tel: +82-41-550-5295, Fax: +82-41-550-5296

E-mail: junhyunoh@smu.ac.kr

(c) 2020, The Korean Society for Microbiology and Biotechnology charides, flavones, microelements and amino acids [2]. Some studies reported that maca comprised of various classes of bioactive compounds such as saponins, alkaloids, steroid hormones, and polyphenol compounds [3]. Dried maca hypocotyls also contain several classes of secondary metabolites, including alkaloids, amino acids, glucosinolates, polysaccharides, fatty acids and macamides [4].

Macamides are secondary amides only found in maca and formed from benzylamine and a fatty acid moiety with varying hydrocarbon chain lengths [5]. Macamides are considered as biomarkers for maca, and the isolation 
and identification of macamides in maca have been studied extensively [5-7]. Xia et al. [8] identified 7 macamides using a HPLC, which were 9-oxo-10E,12Z-octadecadienoicacid, 9-oxo-10E,12E-octadecadienoicacid, $N$ benzyl-9-oxo-10E,12Z-octadecadienamide, $N$-benzyl-9oxo-10E,12E-octadecadienamide, $\quad N$-benzyl-9Z,12Z,15Zoctadecatrienamide, $N$-benzyl-9Z,12Z-octadecadienamide, and $N$-benzyl-hexadecanamide. Among these identified macamides, $N$-benzyl-hexadecanamide (macamide B) was one of the major macamides and frequently served as a biomarker for maca [7, 8].

Owing to its chemical components, maca is known to possess various health-improving and beneficial effects on fertility, energy, memory, osteoporosis, prostate, and skin [9]. It is commonly claimed by the nutrition industry that maca has the ability to improve energy efficiency and modulate the response against oxidative stress [10, 11]. Berlowski et al. [10] reported that maca played an important role as an antioxidant by radical scavenging or by maintaining intracellular ATP production in conditions of oxidative stress. The polysaccharides present in maca root also exhibited strong antioxidant activities by scavenging capacities on hydroxyl, DPPH, and superoxide anion radicals [11]. Recently, Wang and Zhu [12] summarized the health effects of maca; beneficial effects on male and female reproductive systems, antifatigue, antioxidation, neuroprotection, anticancer, hepatoprotection, antimicrobial and pesticidal activity, immune-regulation, prevention against ultraviolet radiation and so on. Rodríguez-Huamán et al. [13] reported that maca increased cell viability and decreased cell cytotoxicity in PC12 cells. Maca also exhibited inhibitory activity against the proliferation of the HT-29 cancer cell line with $\mathrm{IC}_{50}$ of $12.8 \mu \mathrm{mol} / /$ [13]. Choi et al. [14] reported that lipid-soluble extract from maca powder improved swimming endurance capacity in mice. The anti-fatigue effect of maca was evaluated by the forced swimming test using different mice models, including male Kunming mice and ICR mice [11]. Dried yellow maca root powder, macamides, polypeptides and polysaccharides could be used for anti-fatigue purpose [15]. Further research showed that maca root powder at a dose of $400 \mathrm{mg} / \mathrm{kg} / \mathrm{d}$ for 30 days prolonged swimming duration, increased liver glycogen content, and decreased blood lactic acid of male Kunming mice [16].

Ultrasonification-assisted (UA) extraction has gained considerable attention due to its positive influence on heat and mass transfer, resulting in an efficient extraction of bioactive compounds in a shorter time and lower temperatures than traditional methods [17, 18]. UA extraction has been considered as a useful technique to recover, identify, and quantify alkaloid components from medicinal and food materials [17]. Chen et al. [9] optimized the UA extraction process to extract and identify macamides from maca at some conditions; solid/ solvent ratio of $1: 10$, extraction temperature of $40^{\circ} \mathrm{C}$, extraction time of $30 \mathrm{~min}$, and extraction power of $200 \mathrm{~W}$.

This study aims to evaluate the efficacy of UA extraction on the functionality of maca root as compared to the conventional extraction methods such as hot water and ethanol extraction. The specific objectives of this research are to 1) compare the antioxidant activities among the maca extracts, 2) determine macamide B contents present in maca extracts, and 3) evaluate the in vitro effects of maca extracts on the cell viability and creatine kinase activity.

\section{Materials and Methods}

\section{Extraction of maca}

The maca roots cultivated from Peru was used for this study. The dried maca roots were ground and stored in a freezer until use. The ground maca powder (12.5 g) was mixed with either $125 \mathrm{ml}$ of distilled water or $75 \%$ (v/v) ethanol and maca extract was obtained using a soxhlet extractor at $100^{\circ} \mathrm{C}$ for $5 \mathrm{~h}$ (water extract), and at $60^{\circ} \mathrm{C}$ for $3 \mathrm{~h}$ (ethanol extract). For UA extraction, the maca powder ( $2.5 \mathrm{~g}$ ) was placed in $25 \mathrm{ml}$ of $75 \%$ (v/v) ethanol. The extraction was performed in an ultrasonic bath (Asia Ultrasonic, Co., Korea) operated at $60 \mathrm{kHz}$ and $400 \mathrm{~W}$ for $1 \mathrm{~h}$ at room temperature. After UA extraction, the aqueous extract was filtered, concentrated in a vacuum rotary evaporator (Eyela, Rikakikai Co., China), and freeze-dried prior to further use.

\section{Total phenolic content}

The total phenolic content of the maca extracts was determined using the Folin-Ciocalteu method with minor modifications. A portion of $0.02 \mathrm{ml}$ of the diluted extracts was mixed with $0.1 \mathrm{ml}$ Folin-Ciocaleu reagent (Sigma Co., USA), followed by the addition of $0.3 \mathrm{ml}$ 
sodium carbonate ( $20 \%$ aqueous solution). The mixture was kept in the dark for $2 \mathrm{~h}$, and the absorbance was measured at $765 \mathrm{~nm}$ using a spectrophotometer (VIS/UV mini 1240, Shimadzu, Japan). The total phenolic content of the extracts $/ \mathrm{mg}$ ) was expressed as gallic acid equivalents (GAE, $\mu \mathrm{g} / \mathrm{mg})$ [19].

\section{Flavonoid content}

An aliquot of $1 \mathrm{ml}$ of maca extract $(1 \mathrm{mg} / \mathrm{ml})$ was added to $1 \mathrm{ml}$ of $2 \%$ aluminum chloride methanolic solution, allowed to stand for $15 \mathrm{~min}$, and the absorbance was measured at $430 \mathrm{~nm}$. The standard curve was constructed using quercetin $(6.25-100 \mu \mathrm{g} / \mathrm{ml})$ (Sigma Co.). The total flavonoid content $(\mathrm{mg} / \mathrm{ml})$ in the samples was expressed as quercetin equivalent (QE, $\mathrm{mg} / \mathrm{ml}$ ) [20].

\section{Determination of DPPH radical scavenging activity}

The free radical scavenging activity was measured using 2, 2-diphenyl-1-picrylhydrazyl (DPPH) as described by Brand-Williams [21]. Ascorbic acid (Sigma Co.) was used as positive control. After taking $100 \mu \mathrm{l}$ of the diluted sample into a 96-well plate, $100 \mu \mathrm{l}$ of DPPH reagent (Sigma Co.) was added into the wells. The mixture was placed at room temperature under dark condition for $30 \mathrm{~min}$. Then, the absorbance of mixture solution was measured at $520 \mathrm{~nm}$ using a plate reader (Synergy H1, Bio Tec, USA). The radical scavenging activity was determined by following equation: DPPH radical scavenging activity $(\%)=(($ Absorbance of the control - Absorbance of the sample)/Absorbance of the control) $\times 100$ [22].

\section{Ferric reducing antioxidant power (FRAP)}

The FRAP assay was performed according to Lee and Chang [23]. The FRAP working solution contained $1 \mathrm{ml}$ of $10 \mathrm{mmol}$ neocuproine 2, 4, 6-tripyridyl-s-triazine (TPTZ) solution (dissolved in $40 \mathrm{mmol}$ hydrochloric acid), $1 \mathrm{ml}$ of $20 \mathrm{mmol}$ ferric chloride solution, and $10 \mathrm{ml}$ of the acetate buffer solution ( $\mathrm{pH}$ 3.6). A mixture of $60 \mu \mathrm{l}$ of maca extracts and $180 \mu \mathrm{l}$ of the FRAP solution was allowed to react for 5 min under the dark condition. The absorbance of the colored product of the ferrous tripyridyltriazine complex was determined at $593 \mathrm{~nm}$ using a plate reader (Synergy H1). The FRAP of the maca extract was calculated from the Trolox standard curve and expressed as Trolox equivalent (TE, $\mathrm{mg} / \mathrm{g}$ ) [24].

\section{HPLC analysis for macamide B}

One gram of ground maca powder was extracted with $40 \mathrm{ml}$ of ethyl ether in an ultrasonicator (Asia Ultrasonic, Co.) operated at $400 \mathrm{~W}$ for $15 \mathrm{~min}$ at $50^{\circ} \mathrm{C}$. After centrifugation at 5,000 rpm for $10 \mathrm{~min}$, the supernatant was concentrated to dryness at $50^{\circ} \mathrm{C}$ by a rotary evaporator (Eyela, Rikakikai Co.). The residue was made up to exactly $10 \mathrm{ml}$ with acetonitrile using a volumetric flask. The sample was finally filtered through a $0.22 \mu \mathrm{m}$ filter prior to HPLC analysis.

The HPLC analysis for macamide B was performed on a Shimadzu LC-20AD XR consisting of an auto sampler and binary pump system (Shimadzu) coupled with a photodiode array (PDA) detector (SPD-M20A). An aliquot of $10 \mu \mathrm{l}$ of sample solution was injected and analyzed using a Agilent Poraris 5 C18-A $5 \mu \mathrm{m}(250 \mathrm{~mm} \times 4.6 \mathrm{~mm})$ column (Agilent Technologies, USA) with an isocratic elution (acetonitrile:water:formic acid, 90:9.98:0.02) for $30 \mathrm{~min}$. The flow rate was set at $0.6 \mathrm{ml} / \mathrm{min}$, and the column temperature was $30^{\circ} \mathrm{C}$. HPLC chromatograms of macamide $B$ were recorded at $210 \mathrm{~nm}$ [6]. The macamide B standard ( $N$-benzyl-hexadecanamide, Cat\# CFN90843) was purchased from Sigma Co.

\section{Cell culture and cytotoxicity test}

The skeletal muscle cell line $\mathrm{C} 2 \mathrm{C} 12$ myoblasts (CRL1772, ATCC, USA) were maintained in high glucose Dulbecco's Modified Eagle Medium (DMEM) supplemented with 10\% FBS and 1\% penicillin solution and were cultured at $37^{\circ} \mathrm{C}$ in $5 \% \mathrm{CO}_{2}$ condition. $\mathrm{C} 2 \mathrm{C} 12$ myoblasts were seeded into 96 -well plate $\left(1 \times 10^{4} \mathrm{cell} /\right.$ well $)$ for $24 \mathrm{~h}$ and the medium was changed to $0.01-5 \mathrm{mg} / \mathrm{ml}$ concentration of various maca samples for an additional $24 \mathrm{~h}$. After the sample treatment, $10 \mathrm{\mu l}$ of MTT 3-(4,5dimethylthiazol-2-yl)-2,5-diphenyltetrazolium bromide, a tetrazole) (MTT) (M5655, Sigma Co.) solution ( $5 \mathrm{mg} /$ $\mathrm{ml}$ ) was added to each well for $3 \mathrm{~h}$ to evaluate the cytotoxicity of $\mathrm{C} 2 \mathrm{C} 12$ myoblasts. The formazan precipitates were dissolved in $100 \mu \mathrm{l}$ of DMSO (D0458, Samchun Chemicals, Korea) and the absorbance was measured at $595 \mathrm{~nm}[25]$.

\section{Cytoprotective effects of maca extract}

The $\mathrm{C} 2 \mathrm{C} 12$ myoblasts were seeded into 96-well plate $\left(1 \times 10^{4} \mathrm{cell} / \mathrm{well}\right)$ and cultured for $24 \mathrm{~h}$. Then, fresh hydrogen peroxide $\left(\mathrm{H}_{2} \mathrm{O}_{2}\right)(\mathrm{H} 1009$, Sigma Co.) solution 
(final concentration of $0.5 \mathrm{mmol}$ ) and the maca samples (final concentration of $1 \mathrm{mg} / \mathrm{ml}$ ) were co-treated for another $24 \mathrm{~h}$. Then, $10 \mu \mathrm{l}$ of MTT solution $(5 \mathrm{mg} / \mathrm{ml})$ was added to each well for $3 \mathrm{~h}$ to evaluate the cytoprotective effects of maca samples. The formazan precipitates were dissolved in $100 \mu \mathrm{l}$ of DMSO and the absorbance was measured at $595 \mathrm{~nm}$ [26].

\section{Creatine kinase activity}

The creatine kinase (CK) activity was examined by commercial kits (ab155901, ABCAM, UK). Briefly, C2C12 myoblasts were seeded $\left(2.0 \times 10^{6}\right.$ cell/well $)$ into six-well plates and cultured for $24 \mathrm{~h}$, then the $\mathrm{H}_{2} \mathrm{O}_{2}$ solution (final concentration, $0.5 \mathrm{mmol}$ ) and maca samples (final concentration, $1 \mathrm{mg} / \mathrm{ml}$ ) were co-treated for another $24 \mathrm{~h}$. The $\mathrm{C} 2 \mathrm{C} 12$ myoblasts were washed twice by cold Phosphate-buffered saline (PBS) and homogenized in CK assay buffer, centrifuged to remove insoluble materials and the supernatant was diluted and combined with reaction mix to read the OD value at 450 $\mathrm{nm}$ in kinetics mode for $60 \mathrm{~min}$.

\section{Statistical analysis}

Each experiment was repeated three times. One way analysis of variance (ANOVA) was performed using SPSS software (version 11.5, SPSS Ins., USA). Differences among means were analyzed using Duncan's Multiple Range Test (DMRT), and the significance level was defined at $p<0.05$.

\section{Results and Discussion}

\section{Total phenolic content}

The total phenolic content (TPC) of water, ethanol, and ultrasonification-assisted (UA) extracts of maca roots are presented in Fig. 1. The TPC was determined as $1.94,2.53$, and $2.90 \mathrm{GAE} \mu \mathrm{g} / \mathrm{mg}$ for water, ethanol, and UA extracts of maca roots, respectively. This result is in a good agreement with the report that the total polyphenolic contents from maca extracts ranged from 0.09 to $3.29 \mathrm{mg} / \mathrm{ml}$ depending on the extraction solvents [27].

There are considerable reports that ultrasonification treatment significantly can increase the extraction yields and polyphenolic compounds from natural plants $[17,18,28]$. As compared to water stirrer extraction, the

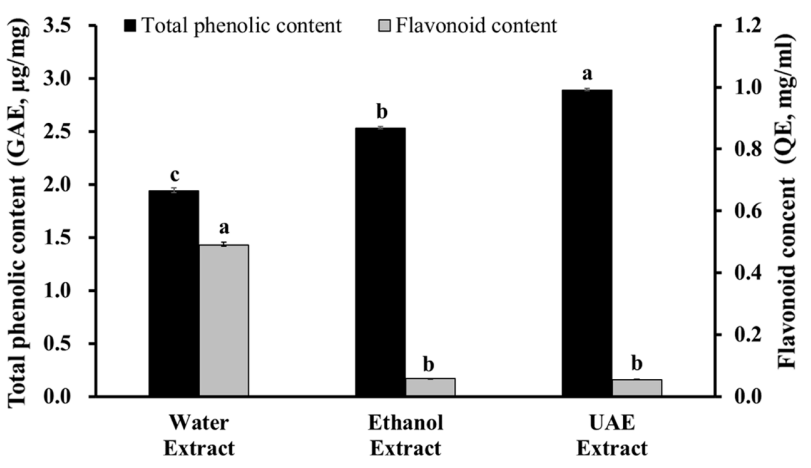

Fig. 1. Total phenolic content and flavonoid content of maca extracts. GAE and QE indicate gallic acid equivalent and quercetin equivalent, respectively. UA indicates ultrasonificationassisted. Error bars represent standard deviation $(n=3)$. Different letters $(a, b)$ represent significant differences among the samples at $p<0.05$.

ultrasonification treatment for $3 \mathrm{~h}$ at $60^{\circ} \mathrm{C}$ with $70 \%$ ethanol resulted in a $104.03 \%$ increase in TPC of Crataegus pinnatifida Bunge fruit [28]. In this research, the UA extract of maca roots also resulted in a significant increase in the TPC $(p<0.05)$.

In general, the extracts containing higher TPCs also exhibited higher flavonoid contents [28]. However in this study, the ethanol and UA extracts containing higher TPCs exhibited lower flavonoid content than the water extract. Flavonoids are part of polyphenols and include anthocyanindins, flavanols, flavones, flavonols, flavonones and isoflavonones. The discrepancy between the TPCs and flavonoid contents in maca roots may be attributed to the different individual phenolics and flavonoid compounds present in maca root. The ultrasonification treatment did not affect the flavonoid content in maca extract, thereby exhibiting no statistical difference in flavonoid contents between the ethanol extract and UA extract.

\section{DPPH radical scavenging activity}

DPPH radical scavenging activity is a common indicator to evaluate the antioxidant activity of natural extracts. The antioxidant activity of primary antioxidants can be assessed by evaluating the hydrogen donating ability to free radicals, resulting in conversion of harmful free radicals to non-toxic species during propagation steps of lipid oxidation [15]. The DPPH radical scavenging activities of water, ethanol, and UA extracts were $38.8 \%, 40.6 \%$ and 41.8\%, respectively. Park et al. 


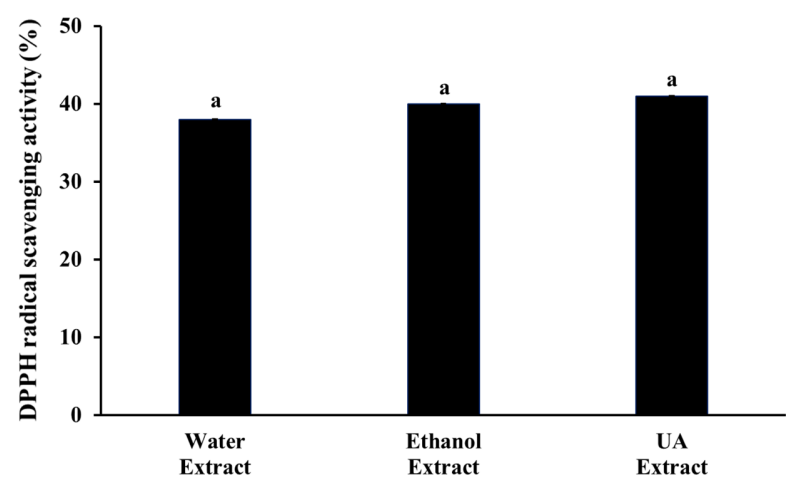

Fig. 2. DPPH radical scavenging activity of maca extracts. UA indicates ultrasonification-assisted. Error bars represent standard deviation $(n=3)$. The superscript letter (a) represents significant differences among the samples at $p<0.05$.

[29] also reported that the DPPH radical scavenging activity of $70 \%$ ethanol extracts obtained from maca root was determined as approximately $43 \%$. In their report, the free radical scavenging activities of maca root exhibited higher activity than maca leaves, and the activity was significantly dose dependent. Sandoval et al. [30] reported that the DPPH radical scavenging activity of maca roots were ranged from $21.64 \%$ to $71.38 \%$ depending on the concentration of maca extracts ( 0.03 to 0.3 $\mathrm{mg} / \mathrm{ml})$.

The DPPH radical scavenging activity in this study did not exhibit any statistical difference among the extracts. The statistical indifference of DPPH radical scavenging activity among the treatments in this study may be attributed to the TPCs among the treatments. There are some reports regarding the compounds responsible for antioxidant activity of maca extract [31, 32]. Gan et al. [31] reported that phenols and alkaloids were the most important substances for antioxidant activity of maca, of which the antioxidant effect of alkaloids seemed to be higher than that of phenols. In addition, Caicai et al. [32] studied two fractions of polysaccharides (MLP-1 and MLP-2) and demonstrated their antioxidant capability.

\section{FRAP}

The FRAP method is a method using the principle of reducing $\mathrm{Fe}^{3+}$ to $\mathrm{Fe}^{2+}$ by phenolic compounds as antioxidants. Therefore, FRAP provides a direct estimate of antioxidant activities or reductants present in the extracts, and also the ability of the analytes to reduce

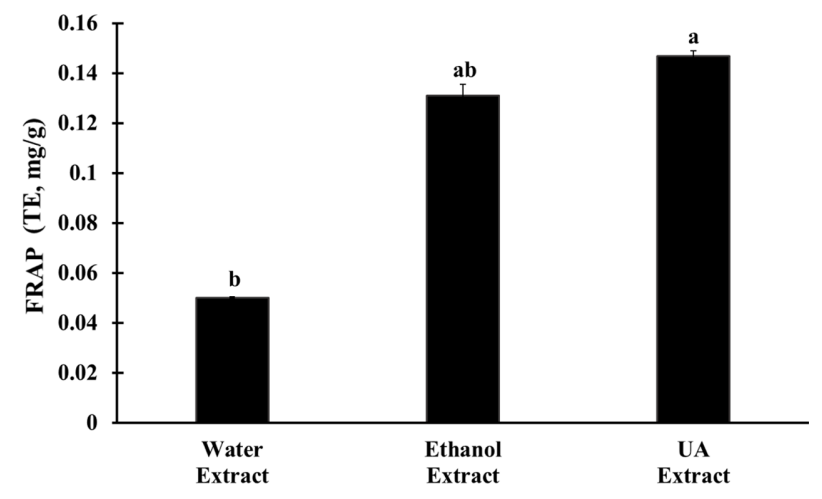

Fig. 3. Ferric reducing antioxidant power of maca extracts. UA indicates ultrasonification-assisted. Error bars represent standard deviation $(n=3)$. Different letters $(a, b)$ represent significant differences among the samples at $p<0.05$.

the $\mathrm{Fe}^{3+} / \mathrm{Fe}^{2+}$ pair. The ethanol and UA extracts of maca root exhibited significantly higher FRAP activities than the water extract $(p<0.05)$ (Fig. 3). This result exhibited almost similar trend with the total phenolic contents of maca extracts. Gan et al. [31] also reported that the TPCs exhibited significant linear correlations to FRAP in maca extracts. There was no statistical difference in FRAP between the ethanol and UA extracts.

Overall, the antioxidant activity of maca extracts determined in this study was comparable with other literatures. The degree of antioxidant activity of maca extracts was mainly dependent on the extraction solvents, and the ultrasonification treatment did not affect the antioxidant activity of maca roots significantly.

\section{Macamide B content}

Macamides are believed to be found only in maca and considered as the bioactive marker compounds in maca [8]. Among the identified macamides, $N$-benzyl9Z,12Z,15Z-octadecatrienamide, $N$-benzyl-9Z,12Z-octadecadienamide, and $N$-benzyl-hexadecanamide (macamide B) were the major macamide compounds [6]. Therefore, macamide B was selected as a biomarker in this study. Fig. 4. presents the HPLC chromatograms of macamide $B$ standard and macamide $B$ present in water, ethanol, and UA extracts of maca root. The retention time of macamide B of standard was determined as $18.872 \mathrm{~min}$.

The calculated macamide B contents of water, ethanol, and UA maca root extracts are presented in Table 1. Macamide B was not detected in water extract, presum- 


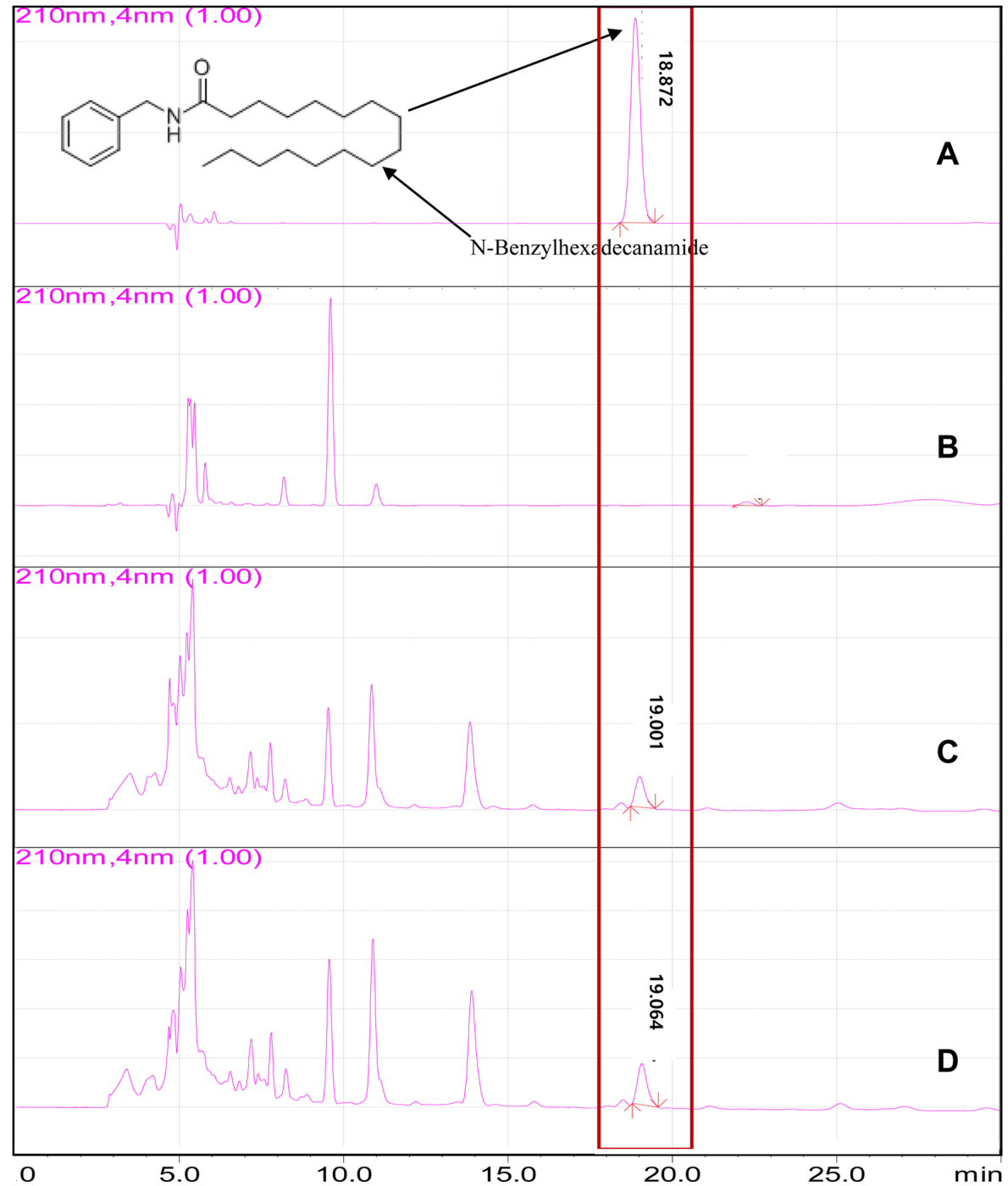

Fig. 4. HPLC chromatograms of (A) $\mathbf{N}$-benzylhexadecanamide (macamide B) standard, (B) macamide B present in water extract, (C) ethanol extract, and (D) ultrasonification-assisted extract (D).

able due to insolubility of macamide $B$ in water. Macamide B has a long chain fatty acid and $N$-benzylamide,

Table 1. Macamide B contents of maca extracts analyzed by HPLC.

\begin{tabular}{cccc}
\hline Maca extract & $\begin{array}{c}\text { Retention } \\
\text { time } \\
(\mathrm{min})\end{array}$ & Area & $\begin{array}{c}\text { Macamide B } \\
\text { content } \\
(\mu \mathrm{g} / \mathrm{mg})\end{array}$ \\
\hline Water extract & - & - & - \\
$\begin{array}{c}\text { Ethanol extract } \\
\text { Ultrasonification- } \\
\text { assisted extract }\end{array}$ & 19.001 & 179142 & $0.087 \pm 0.142$ \\
\hline
\end{tabular}

-, indicates "not detected". resulting in sparingly solubility in polar solvent such as water. The macamide $\mathrm{B}$ contents of ethanol and UA maca root extracts were determined as 0.087 and 0.083 $\mu \mathrm{g} / \mathrm{mg}$, respectively. This result is in a good agreement with the report that the macamide $\mathrm{B}$ contents in maca were determined from 0.095 to $0.194 \mathrm{mg} / \mathrm{g}$ [12]. There was no significant difference in macamide B contents between the ethanol and UA maca root extracts.

\section{Cytotoxicity test}

In the cytotoxicity test with the skeletal muscle cell, all of the maca extracts exhibited a dose-dependent pattern as the concentration increased from 0.01 to $5 \mathrm{mg} / \mathrm{ml}$ 


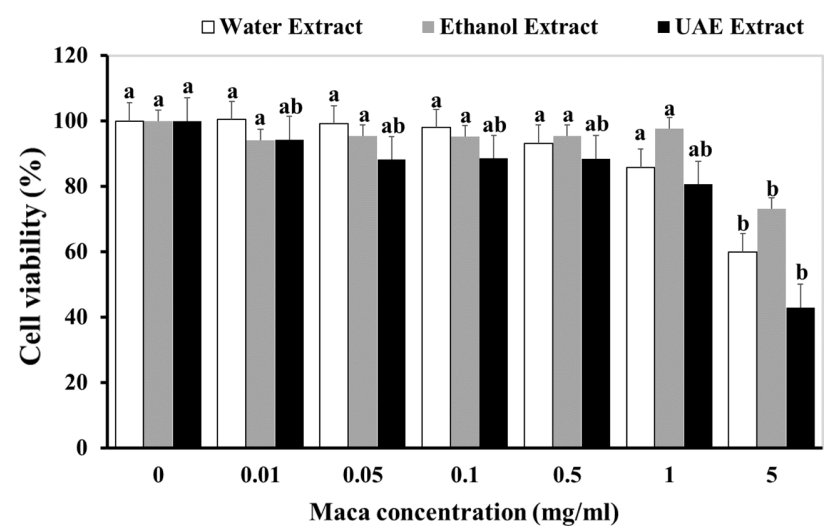

Fig. 5. Cell viability of maca extracts. UA indicates ultrasonification-assisted. Error bars represent standard deviation $(n=3)$. Different letters $(a, b)$ represent significant differences among the samples at $p<0.05$.

(Fig. 5). The maca extracts maintained greater than $80 \%$ cell viability up to the concentration of $1 \mathrm{mg} / \mathrm{ml}$. However cell viability at the concentration of $5 \mathrm{mg} / \mathrm{ml}$ was significantly decreased, indicating that the maca extract at concentrations up to $1 \mathrm{mg} / \mathrm{ml}$ would not affect the cell viability. There was no significant difference in cell viability among the water, ethanol, and UA maca root extracts. Therefore, the concentration of $1 \mathrm{mg} / \mathrm{ml}$ was determined to be used for further test toward the muscle cell.

\section{Cytoprotective effects of maca extract}

Oxidative stress is caused by increment of cellular reactive oxygen species (ROS) level. $\mathrm{H}_{2} \mathrm{O}_{2}$ treatment in cell line can increase ROS level, thereby inducing oxidative damage and cell death [33-35]. To investigate the cytoprotective effects of the maca extracts, $\mathrm{H}_{2} \mathrm{O}_{2}$ was treated with $\mathrm{C} 2 \mathrm{C} 12$ myoblasts to induce oxidative stress.

As estimated by the MTT assay, the $\mathrm{H}_{2} \mathrm{O}_{2}$-treated control group induced approximately $30 \%$ of cytotoxicity as compared with the normal control group (no $\mathrm{H}_{2} \mathrm{O}_{2}$ treatment) (Fig. 6). However, the treatment of the cell with the maca extracts induced less reduction of cell viability than the $\mathrm{H}_{2} \mathrm{O}_{2}$-treated control group. The results indicated that the water and UA maca root extracts exhibited higher cytoprotective effects in oxidative stress condition than the ethanol extract. RodríguezHuamán [13] reported that $10 \mu \mathrm{g}$ of maca methanol extract increased the cell viability of $\mathrm{PC} 12$ cell by $31 \%$ at

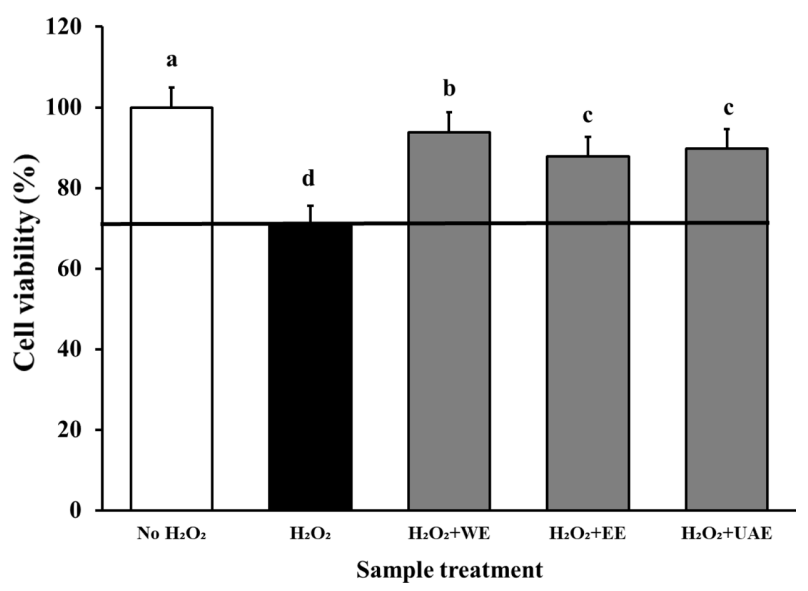

Fig. 6. Cell viability of maca extracts in the presence of hydrogen peroxide. WE, EE and UAE indicate water extract, ethanol extract, and ultrasonification-assisted extract, respectively. Error bars represent standard deviation $(n=3)$. Different letters $(a, b, c, d)$ represent significant differences among the samples at $p<0.05$.

6 h-pretreatment of the extract. Their result indicated that the methanol extraction of maca confirmed the antioxidant capacity of maca.

\section{Effects of maca extracts on creatine kinase activity}

Creatine kinase (CK) converts creatine into phosphocreatine and ADP to maintain energy homeostasis during muscle contraction [36]. The level of CK was evaluated after $\mathrm{H}_{2} \mathrm{O}_{2}$ and sample treatment. The $\mathrm{H}_{2} \mathrm{O}_{2}$ -

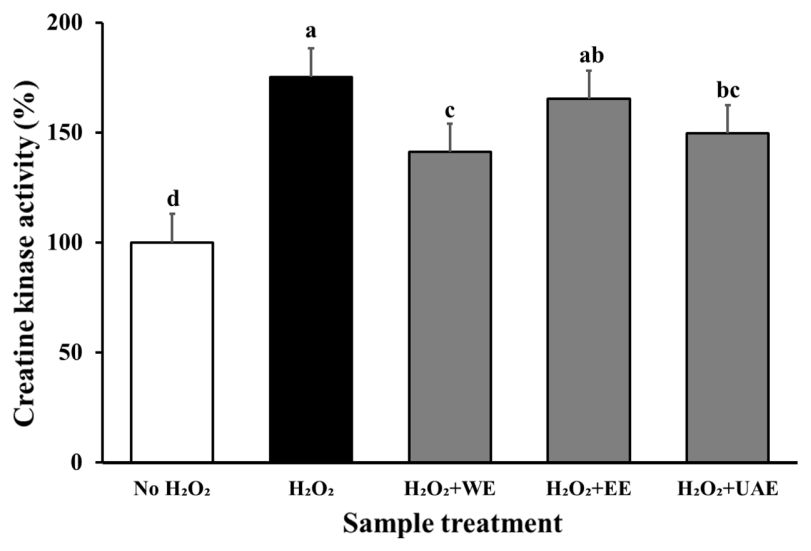

Fig. 7. Creatine kinase activity of maca extracts in the presence of hydrogen peroxide. WE, EE and UAE indicate water extract, ethanol extract, ultrasonification-assisted extract, respectively. Error bars represent standard deviation $(n=3)$. Different letters $(a, b, c, d)$ represent significant differences among the samples at $p<0.05$. 
treated control group exhibited significantly higher CK level than the normal control group (Fig. 7). The treatments with the water extract and UA extracts resulted in significantly lower $\mathrm{CK}$ level than the $\mathrm{H}_{2} \mathrm{O}_{2}$-treated control group, which indicated the cell protective effects under the oxidative stress conditions.

Increased CK level indicates the cell damage and symptoms of fatigue which is activated and released in muscle through Phosphocreatine $(\mathrm{PCr})$ system under the oxidative stress conditions [37]. In the previous studies, blood CK levels were significantly inhibited by various sample treatments in exercise-induced fatigue [3841]. Furthermore, several in vivo studies demonstrated the anti-fatigue effects of maca treatment in mice [4244]. Therefore, the results in this study suggest that maca treatment can play significant roles in muscle protection and anti-fatigue under oxidative stress condition in cellular levels.

\section{Acknowledgments}

This research was financially supported by the Ministry of Small and Medium-sized Enterprises (SMEs) and Startups (MSS), Korea, under the "Regional Specialized Industry Development Program (R\&D, P0006422)" supervised by the Korea Institute for Advancement of Technology (KIAT).

\section{Conflict of interest}

The authors have no financial conflicts of interest to declare.

\section{References}

1. Chen L, Li J, Fan L. 2017. The nutritional composition of maca in hypocotyls (Lepidium meyenii Walp.) cultivated in different regions of China. J. Food Qual. 2017: 1-8.

2. Zhou Y, Li P, Brantner A, Wang H, Shu X, Yang J, et al. 2017. Chemical profiling analysis of Maca using UHPLC-ESI-Orbitrap MS coupled with UHPLC-ESI-QqQ MS and the neuroprotective study on its active ingredients. Sci. Rep. 7: 44660.

3. Tang W, Jin L, Xie L, Huang J, Wang N, Chu B, et al. 2017. Structural characterization and antifatigue effect in vivo of maca (Lepidium meyenii Walp) polysaccharide. J. Food Sci. 82: 757-764.

4. McCollom MM, Villinski JR, McPhail KL, Craker LE, Gafner S. 2005. Analysis of macamides in samples of Maca (Lepidium meyenii) by HPLC-UV-MS/MS. Phytochem. Anal. 16: 463-469.

5. Zhang L, Li G, Wang S, Yao W, Zhu F. 2017. Physicochemical properties of maca starch. Food Chem. 218: 56-63.

6. Chen J-J, Zhao Q-S, Liu Y-I, Gong P-F, Cao L-L, Wang X-D, et al.
2017. Macamides present in the commercial maca (Lepidium meyenii) products and the macamide biosynthesis affected by postharvest conditions. Int. J. Food Prop. 20: 3112-3123.

7. Chain FE, Grau A, Martins J, Catalán CAN. 2014. Macamides from wild Maca, Lepidium meyenii Walpers (Brassicaceae). Phytochem. Lett. 8: 145-148.

8. Xia C, Deng J, Chen J, Zhu Y, Song Y, Zhang Y, et al. 2019. Simultaneous determination of macaenes and macamides in maca using an HPLC method and analysis using a chemometric method (HCA) to distinguish maca origin. Rev. Bras. Farmacogn. 6: 702-709.

9. Chen S-X, Li K-K, Pubu D, Jiang S-P, Chen B, Chen L-R, et al. 2017. Optimization of ultrasound-assisted extraction, HPLC and UHPLC-Q-TOF-MS/MS analysis of main macamides and macaenes from maca (Cultivars of Lepidium meyenii Walp). Molecules 22: 2196.

10. Berlowski A, Zawada K, Wawer I, Paradowska K. 2013. Antioxidant properties of medicinal plants from Peru. Food Nutr. Sci. 4: 71-77.

11. Li J, Chen L, Li J, Duan Z, Zhu S, Fan L. 2017. The composition analysis of Maca (Lepidium meyenii Walp.) from xinjiang and its antifatigue activity. J. Food Qual. 2017: 2904951.

12. Wang S, Zhu F. 2019. Chemical composition and health effects of maca (Lepidium meyenii). Food Chem. 288: 422-443.

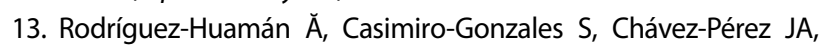
Gonzales-Arimborgo C, Cisneros-Fernández R, Aguilar-Mendoza $L A$, et al. 2016. Antioxidant and neuroprotector effect of Lepidium meyenii (maca) methanol leaf extract against 6-hydroxy dopamine (6-OHDA)-induced toxicity in PC12 cells. Toxicol. Mech. Methods 27: 279-285.

14. Choi EH, Kang Jl, Cho JY, Lee SH, Kim TS, Yeo IH, et al. 2012. Supplementation of standardized lipid-soluble extract from maca (Lepidium meyenii) increases swimming endurance capacity in rats. J. Funct. Foods 4: 568-573.

15. Li S, Hao L, Kang Q, Cui Y, Jiang H, Liu X. 2017. Purification, characterization and biological activities of a polysaccharide from Lepidium meyenii leaves. Int. J. Biol. Macromol. 103: 1302-1310.

16. Tang W, Jin L, Xie L, Huang J, Wang N, Chu B, et al. 2017. Structural characterization and antifatigue effect in vivo of maca (Lepidium meyenii Walp) polysaccharide. J. Food Sci. 82: 757-764.

17. Zhang Y, Liu C, Qi Y, Li S, Pan Y, Li Y. 2015. Circulating ultrasoundassisted extraction, countercurrent chromatography, and liquid chromatography for the simultaneous extraction, isolation, and analysis of the constituents of Uncaria tomentosa. J. Chromatogr. A. 1388: 36-42.

18. Chavan Y, Singhal RS. 2013. Ultrasound-assisted extraction (UAE) of bioactives from arecanut (Areca catechu L.) and optimization study using response surface methodology. Innov. Food Sci. Emerg. 17: 106-113.

19. Lo KM, Cheung PCK. 2005. Antioxidant activity of extracts from the fruiting bodies of Agrocybe aegerita var. alba. Food Chem. 89: 533-539.

20. Hwang ES, Thi ND. 2014. Antioxidant contents and antioxidant activities of hot-water extracts of aronia (Aronia melancocarpa) 
with different drying methods. Korean J. Food Sci. Technol. 46: 303-308.

21. Brand-Williams W, Cuvelier ME, Berset C. 1995. Use of a free radical method to evaluate antioxidant activity. Food Sci. Technol. 28: 25-30.

22. Wang KJ, Zhang YJ, Yang CR. 2005. Antioxidant phenolic compounds from rhizomes of Polygonum paleaceum. J. Ethnopharmacol. 96: 483-487.

23. Lee YK, Chang YH. 2019. Physicochemical and antioxidant properties of methanol extract from Maca (Lepidium meyenii Walp.) leaves and roots. Food Sci. Technol. 39: 278-286.

24. Iris F, Benzie F, Strain JJ. 1996. The ferric reducing ability of plasma (FRAP) as a measure of "antioxidant power": the FRAP assay. Anal. Biochem. 239: 70-76.

25. Lee MH, Jang MH, Kim EK, Han SW, Cho SY, Kim CJ. 2005. Nitric oxide induces apoptosis in mouse $\mathrm{C} 2 \mathrm{C} 12$ myoblast cells. J. Pharmacol. Sci. 97: 369-376.

26. Bosutti A, Degens H. 2015. The impact of resveratrol and hydrogen peroxide on muscle cell plasticity shows a dose-dependent interaction. Sci. Rep. 5: 8093.

27. Kwon Y-S, Jeon In-S, Hwang J-H, Lim D-M, Kang Y-S, Chung H-J. 2009. Biological activities of maca (Lepidium meyenii) extracts. J. Korean Soc. Food Sci. Nutr. 38: 817-823.

28. Park S-J, Kwon S-P, Rha Y-A. 2017. Enhanacement of antioxidant activities of Crataegus pinnatifida bunge fruit by ultrasonification extraction processes. J. Korean Soc. Food Sci. Nutr. 46: 891-895.

29. Park S-J, Kim O-L, Rha Y-A. 2017. Component analysis and antioxidant activity of Maca. Culi. Sci. Hos. Res. 23: 137-144.

30. Sandoval M, Okuhama NN, Angeles FM, Melchor VV, Condezo LA, Lao J, et al. 2002. Antioxidant activity of the cruciferous vegetable Maca (Lepidium meyenii). Food Chem. 79: 207-213.

31. Gan J, Feng Y, He Z, Li X, Zhang H. 2017. Correlations between antioxidant activity and alkaloids and phenols of maca (Lepidium meyenii). J. Food Qual. 2017: 3185945.

32. Caicai K, Limin H, Liming Z, Zhiqiang Z, Yongwu Y. 2018. Isolation, purification and antioxidant activity of polysaccharides from the leaves of maca (Lepidium meyenii). Int. J. Biol. Macromol. 107: 2611-2619.
33. Droge W. 2002. Free radicals in the physiological control of cell function. Physiol. Rev. 82: 47-95.

34. Bergamini CM, Gambetti S, Dondi A, Cervellati C. 2004. Oxygen, reactive oxygen species and tissue damage. Curr. Pharm. Des. 10: 1611-1626.

35. Siu PM, Wang Y, Alway SE. 2009. Apoptotic signaling induced by $\mathrm{H}_{2} \mathrm{O}_{2}$-mediated oxidative stress in differentiated $\mathrm{C} 2 \mathrm{C} 12$ myotubes. Life Sci. 84: 468-481.

36. Perry CG, Heigenhauser GJ, Bonen A, Spriet LL. 2008. High-intensity aerobic interval training increases fat and carbohydrate metabolic capacities in human skeletal muscle. Appl. Physiol. Nutr. Metab. 33: 1112-1123.

37. Ydfors M, Hughes MC, Laham R, Schlattner U, Norrbom J, Perry CG. 2016. Modelling in vivo creatine/phosphocreatine in vitro reveals divergent adaptations in human muscle mitochondrial respiratory control by ADP after acute and chronic exercise. J. Physiol. 594: 3127-3140.

38. An H, Seo S, Sim K, Kim J, Kim E, Lee M, et al. 2006. Antifatigue effect of chlorella vulgaris in mice. Korean J. Food Nutr. 19: 169175.

39. Wang J, Li S, Fan Y, Chen Y, Liu D, Cheng H, et al. 2010. Antifatigue activity of the water-soluble polysaccharides isolated from Panax ginseng CA Meyer. J. Ethnopharmacol. 130: 421-423.

40. Xu C, Lv J, Lo YM, Cui SW, Hu X, Fan M. 2013. Effects of oat $\beta$ glucan on endurance exercise and its anti-fatigue properties in trained rats. Carbohydr. Polym. 92: 1159-1165.

41. Huang Y, Wang Y, Li W, Zhan J, Lei J, Li N, et al. 2019. Evaluation of anti-fatigue property of Porphyridium cruentum in mice. Trop. J. Pharm. Res. 18: 579-584.

42. Yang Q, Jin W, Lv X, Dai P, Ao Y, Wu M, et al. 2016. Effects of macamides on endurance capacity and anti-fatigue property in prolonged swimming mice. Pharm. Biol. 54: 827-834.

43. Li J, Sun Q, Meng Q, Wang L, Xiong W, Zhang L. 2017. Anti-fatigue activity of polysaccharide fractions from Lepidium meyenii Walp (maca). Int. J. Biol. Macromol. 95: 1305-1311.

44. Tang W, Jin L, Xie L, Huang J, Wang N, Chu B, et al. 2017. Structural characterization and antifatigue effect in vivo of maca (Lepidium meyenii Walp) polysaccharide. J. Food Sci. 82: 757-764. 